\title{
Periodontal diseases as bacterial infection
}

\author{
BASCONES MARTINEZ A* \\ FIGUERO RUIZ E**
}

Bascones Martínez A, Figuero Ruiz E. Periodontal diseases as bacterial infection. Av Periodon Implantol. 2005; 17, 3: 111-118.

\begin{abstract}
The periodontal disease is conformed by a group of illnesses affecting the gums and dental support structures. They are caused by certain bacteria found in the bacterial plaque. These bacteria are essential to the onset of illness; however, there are predisposing factors in both the host and the microorganisms that will have an effect on the pathogenesis of the illness. Periodontopathogenic bacterial microbiota is needed, but by itself, it is not enough to cause the illness, requiring the presence of a susceptible host. These diseases have been classified as gingivitis, when limited to the gums, and periodontitis, when they spread to deeper tissues. Classification of periodontal disease has varied over the years. The one used in this work was approved at the International Workshop for a Classification of Periodontal Diseases and Conditions, held in 1999. This study is an overview of the different periodontal disease syndromes. Later, the systematic use of antibiotic treatment consisting of amoxicillin, amoxicillinclavulanic acid, and metronidazole as first line coadjuvant treatment of these illnesses will be reviewed.
\end{abstract}

\section{KEY WORDS}

Classification, periodontal illnesses, biofilm

Publicado en Medicina y Patología Oral 2004; 9 Suppl:S92-107

Acepted for publication: April 2005

\section{INTRODUCTION}

The term infection is used to refer to the presence and multiplication of microorganisms in the body (1). Periodontal disease is a group of illnesses located in the gums and dental support structures (ligament and alveolar bone) and are produced by certain bacteria encountered in subgingival plaque (Fig.1). The most important and most prevalent anaerobic gram-negative bacteria in the subgingival area are Actinobaci-llus actinomycetemcomitans (Aa), Porphyromonas gingiva- lis $(\mathrm{Pg})$, Prevotella intermedia $(\mathrm{Pi})$, and Tannerella forsythensis (Tf). These bacteria play an important role in the onset and subsequent development of periodontitis, participating in the formation of the periodontal pocket, connective tissue destruction, and alveolar bone resorption by means of an immunopathogenic mechanism. Once periodontitis has been established, an inflammatory infiltrate is formed consisting of different kinds of cells, such as macrophages and lymphocytes that will produce different cytokine subtypes, biological mediators responsible for the immunopathology of different illnesses (Table 1)

* Catedrático de Medicina Bucal y Periodoncia. Departamento de Estomatología III. Facultad de Odontología. Universidad Complutense de Madrid.

** Licenciada en Odontología. Becaria del Programa Nacional de Formación de Profesorado Universitario del Ministerio de Educación, Cultura y Deporte. Alumna de doctorado. 


\begin{tabular}{|ll|}
\hline \multicolumn{2}{|c|}{ TABLE 1.- INFECTIONS } \\
Polymicrobial & (3-6 species) \\
Mixtas & (aerobic, facultative, anaerobic) \\
Non specific & $\begin{array}{l}\text { The same clinical manifestations, } \\
\text { different aetiology }\end{array}$ \\
& Dental plaque flora \\
\hline
\end{tabular}

(Fig.2). Most naturally occurring microorganisms grow on the surfaces in the form of biofilm; dental plaque is a good example of this. It is currently known that the phenotype expressed by the bacteria when they grow on a surface differs from the phenotype they express when they grow planctonically. This is of great clinical relevance, particularly due to the increase in biofilm resistance to antimicrobial agents $(2,3)$.

Biofilm is formed in several stages, beginning with the adsorption of host and bacterial molecules onto the tooth surface in order to form the so-called acquired film, which allows the microorganisms that have been passively transported there to interact by means of the forces of attraction of Van der Waals and electrostatic forces of repulsion and attraction, thereby creating a weak bond. This bond is later reinforced by the appearance of strong interactions mediated by specific molecules on the surface of the bacteria (adhesins) with the complementary receptors of the dental film itself. Over time, the phenomena of coaggre-gation of new colonizers and multiplication enable the bacteria to adhere firmly to the dental surface $(2,3)$. The clinical expression of the different periodontitis syndromes will depend on the interaction between host-related factors, the environment and the microbiological agents. A favourable environment plus positive genetic factors will determine an individual_s susceptibility; furthermore the varying degrees of severity of the clinical syndromes, rate of progression, relapse, and the random response to treatment will also play a key role. Periodontopathogenic bacterial microbiota is therefore necessary, but not sufficient on its own for the disease to emerge; a susceptible host must also be present (4). Epidemiological studies have demonstrated a significant association between the severity of periodontal illnesses, the amount of dental plaque and the degree of oral hygiene, with a cause and effect relationship between the formation and accumulation of dental plaque and the development of gingivitis. In this regard, Löe_s studies (5) (1965) on experimental gingivitis conducted in Denmark are important. These studies demonstrated a significant association between the accumulation of bacterial plaque and gingivitis over the 21 days during which the experiment was carried out. The clinical manifestations of gingivitis disappeared when oral hygiene

\section{TABLE 2.- ORAL FLORA CHARACTERISTICS}

HIGHLY COMPLEX

-Heterogeneous

-Multiple species $~ 300$ species

-Different ecosystems

-Abundant

- Specific

-Dynamic

OPPORTUNISTIC

and plaque control methods were reinstated. Subsequently, in another longitudinal study carried out using beagles (dogs), Lindhe (6) (1973) demonstrated experimental periodontitis. Under healthy conditions, the forces of bacterial aggression and host resistance are balanced. When this equilibrium is upset, be it because of an increase in the number and/ or virulence of the germs or because defences are low, the disease emerges. The resulting illnesses have therefore been classified as gingivitis, limited to the gum, and periodontitis, when they spread to underlying tissues, destroying the insertion of connective tissue to the cement and causing pocket formation, alveolar bone resorption, tooth mobility and ultimately leading to the loss of the tooth. When they interact with connective tissue, the bacteria provoke a series of inflammatory and immunological reactions in the host that are translated into an accumulation of cells associated with the activation of periodontal destruction processes. Longitudinal studies suggest that the disease progresses episodically and is characterized by dormant phases followed by exacerbation, resting during the dormant periods and subsequent tissue destruction during active stages. These periods of periodontal destruction are associated with various changes in the cell population that confirms the inflammatory infiltrate located in the subepithelial connective tissue (neutrophiles, macrophages, lymphocytes, plasma cells, etc.) (7). Starting in the 90 s, the hypothesis has been put forth that predisposing factors in the host (such as the lack of oral hygiene, age, systemic factors such as smoking, diabetes, genetic vulnerability, immunological alterations, etc.) play a key role in the pathogenesis of periodontal illness, as well as microbial factors that influence the periodontal pathogenicity of the germs involved (such as specific bacterial adhesion factors). At birth, the oral cavity is sterile, although bacterial colonization quickly begins, creating the socalled oral microbial flora or microbiota, where aerobic, strictly anaerobic (65\%), saprophytic and pathogenic species all coexist. The natural balance (eubiosis) can be upset by exogenous or endogenous factors, thereby leading to disease (dysbiosis). Bacterial plaque located in the gingival margin (supra and subgingival) is what triggers the illness; subgingival plaque is responsible to a greater degree since it has 


\section{TABLE 3.- CLASSIFICATION OF THE WORLD WORISHOP, 1989}

\author{
A. GINGIVITIS \\ a. Dental plaque-induced gingival diseas \\ b. Acute necrotizing ulcerative gingivitis (ANUG). \\ c. Steroid hormone-induced gingivitis. \\ d. Drug-induced gingival enlargements. \\ e. Gingivitis associated with blood disorders, nutritional \\ déficits, tumors, genetic factors, viral infections. \\ f. Gingivitis descamativa.
}

\section{B. PERIODONTITIS}

a. Adult periodontitis.

b. Early-onset periodontitis:

$$
\begin{gathered}
\text { i. Prepuberal periodontitis: } \\
\text { 1.1. Localized } \\
\text { 2.2. Generalized }
\end{gathered}
$$

ii. Juvenile periodontitis

1.1. Localized

2.2. Generalized

c. Periodontitis associated with systemic diseases

d. Necrotising ulcerative periodontitis

e. Refractory periodontitis

greater contact with the tissues that support the tooth. Subgingival plaque is made up of anaerobic, gramnegative bacteria, mobile forms and spirochetes, located in an area with optimal conditions (pockets, anaerobic environments, $\mathrm{Ph}$, oxidoreduction potential, less self-cleaning action, etc). Microbiota is therefore polymicrobial and mixed and the resulting illnesses are often the consequence of complex bacterial associations (Table 2).

\section{CLASSIFICATION OF PERIODONTAL DISEASES}

For many years, the American Association of Periodontics has classified periodontal disease into two categories, i.e., gingivitis and periodontitis (slight, moderate, severe and refractory), according to the area of the gum involved. At the World Workshop in Clinical Periodontics held in 1989, a new classification was established that set itself apart thanks to the incorporation of new descriptive categories (Table 3). Later on, during the course of the First European Workshop on Periodontology (1993) a more straightforward classification of periodontal disease was proposed based largely on the associated causal factors and on the different host response (Table 4). These classifications have been widely used by both clinicians, as well as by researchers, alt-

\section{TABLA 4.- CLASSIFICATION EUROPEAN WORISHOP, 1993}

\author{
A. PRIMARY DESCRIPTORS \\ a. Adult periodontiti \\ b. Early-onset periodontitis \\ c. Necrotising ulcerative periodontitis \\ B. SECONDARY DESCRIPTORS \\ a. Tooth distribution. \\ b. Rate of progression. \\ c. Treatment response. \\ d. Associated with systemic diseases. \\ e. Microbiological characteristics. \\ f. Ethnicity. \\ g. Other factors.
}

hough they present a series of shortcomings. For example, in the classification of the International Workshop, celebrated in 1989, there is an overlap between different categories, gingival disease is conspicuously absent, inappropriate emphasis is placed on the age of onset of the disease, as well on the rates of progression and the presence of inadequate classification criteria. On the other hand, the 1993 European classification lacks details that are needed to properly identify the broad spectrum of periodontal illnesses encountered in clinical practice. In light of all these deficiencies, the 1996 World Workshop on Periodontology underscores the need to revise the existing classifications and to create a new one. In 1997, the American Association of Periodontics decided to set up a committee to take charge of this task; the classification proposed by this committee was approved at the International Workshop for a Classification of Periodontal Diseases and Conditions (1999) (Table 5).

\section{Dental Plaque-Induced Gingival Diseases}

The term "gingival diseases" is used to define the pattern of signs and symptoms of various illnesses located in the gum. They all share the following characteristics: they present bacterial plaque that cause or exacerbate the severity of the lesion; they are reversible if the causative factors are eliminated and they may be the precursor to loss of attachment around the tooth. Clinical manifestations include inflammation of the gum, with elongated gingival contour due to oedema or fibrosis, red or bluish in colour, elevated sulcular temperature, bleeding on probing and increased gingival bleeding. All of these signs are associated with periodontia with stable attachment and without loss of attachment or stable albeit in shrunken periodontia (8) (Fig. 3). Dental plaque-induced is an inflammation of the gum due to the location of bacteria along the gingival margin that 


\section{AVANCES}

Volumen 17 - $\mathrm{N}^{\circ} 3$ - Diciembre 2005

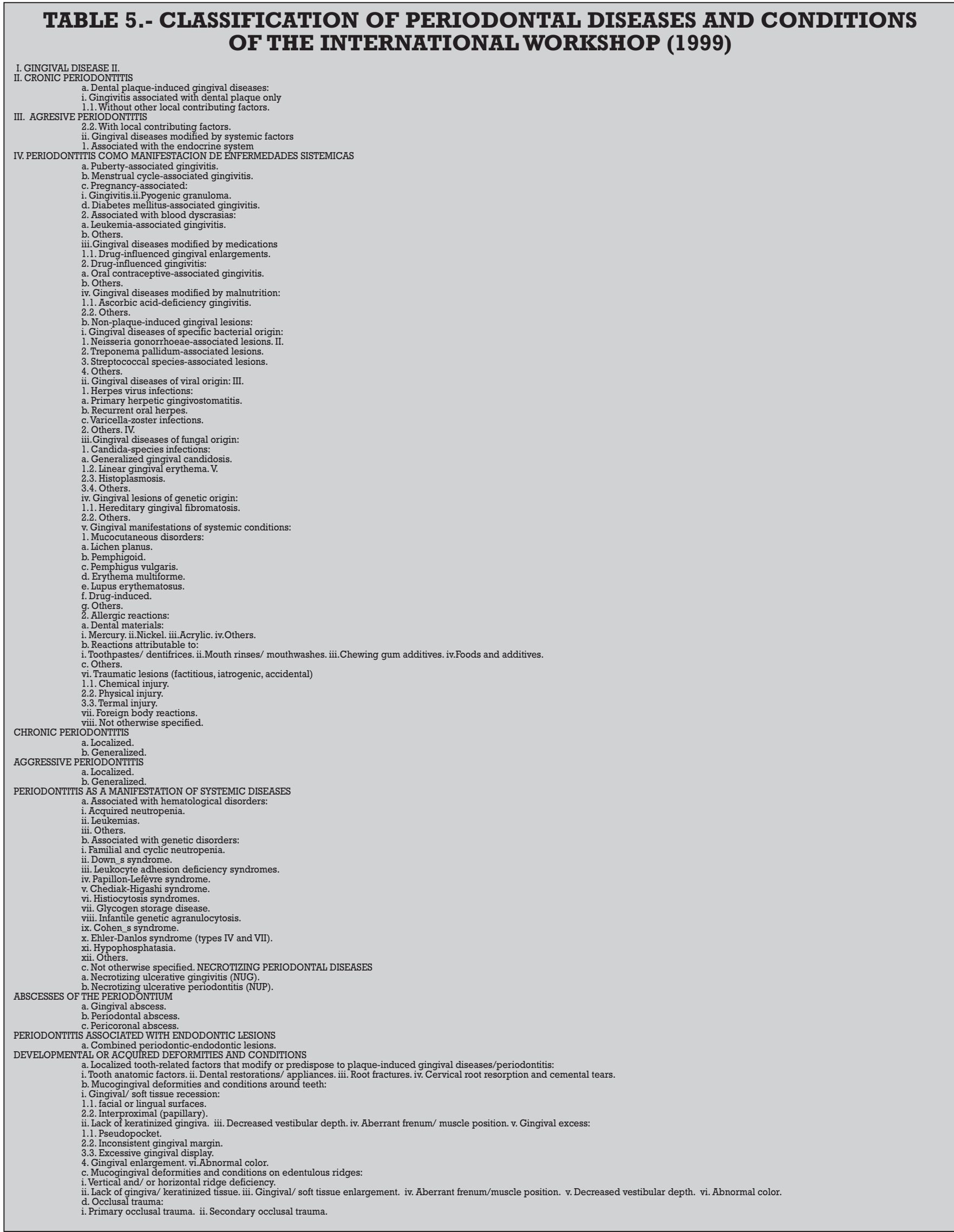


may later spread to the entire gingiva. Characteristic clinical findings include erythema, oedema, bleeding, tenderness and enlargement. The severity of the disease can be influenced by dental anatomy, in addition to the restorative or endodontic status in each case (9) (Fig.1). Gingivitis associated with puberty shares the majority of the clinical signs encountered in plaque-induced gingivitis, but the main distinction between the two has to do with the high propensity to develop overt signs of gingival inflammation with relatively small amounts of bacterial plaque around puberty. During puberty, a host of endocrine changes take place characterised by increased steroid hormone plasma levels that will be responsible for the inflammatory status of the gum (9). The hallmark of gingivitis associated with the menstrual cycle is a moderate inflammatory response of the gum prior to ovulation, with a $20 \%$ increase in the amount of gingival exudate, resulting from the elevation of luteinizing hormone levels $(>25 \mathrm{mU} / \mathrm{ml})$ and/ or estradiol (>200 pg/ ml) (9). Pregnancy-related gingivitis is a proliferative, vascular and nonspecific inflammation of the gingiva with significant inflammatory cell infiltrate. Clinically, it presents as bright red gums that bleed easily, the gingival margin is thickened, and the interdental papilla are hyperplasic which can lead to the appearance of pseudopockets (10). In 1963, Löe and Silness (11) stated that the first symptoms appear in the second month of pregnancy and continue until the eighth month, at which time a certain degree of improvement is seen, before the disease finally stabilizes following delivery. Clinical studies present prevalence rates of between 35 and $100 \%$ (11) of all pregnant women. Granuloma gravidarum, also known as pregnancy tumours, is an exaggerated proliferative fibrovascular inflammatory response to an ordinary stimulus (12) mainly located in the gum. It is described as a nodular or ulcerated localized mass that is red or purplish red, that bleeds easily and that frequently appears in females $(0.5-5 \%)(13,14)$ around the second trimester of pregnancy that grows throughout the pregnancy, reaching sizes that tend to not exceed $2 \mathrm{~cm}$. It is of unknown aetiology, although trauma, hygiene and hormonal factors have been involved (13). In gingivitis associated with diabetes mellitus, diabetic control is of greater relevance than plaque control insofar as the severity of the gingival inflammation is concerned. This type of gingivitis tends to present in children with poorly controlled diabetes mellitus type I. Gingivitis associated with leukaemia is characterised by inflamed, spongy gingival tissues that vary between red and purple in colour. Bleeding is common and may be the first sign of acute leukaemia in $17.7 \%$ and of chronic leukaemia in $4.4 \%$ of the cases, respectively. Gingival enlargement is associated with the use of anticonvulsants (phenytoin), immunosuppressants (cyclosporin A) and calcium channel blockers (nifedipine, verapamil, diltiazem, sodium valproate). Although it varies from one patient to another and even within the same individual, enlarged gums are generally located in the anterior portion of the gingiva, with a greater prevalence in young patients. It tends to appear three months after initiating drug treatment, generally at the level of the papilla and it is not associated with attachment loss (Figs. 4 - 7). Other cases of swollen gums have been observed in association with the use of oral contraceptives, wherein the gingiva are more inflamed in the presence of relatively small amounts of plaque. Malnourished individuals are immunocompromised and this can affect the person_s susceptibility to infection, which would exacerbate the gingival response to the presence of bacterial plaque. The most widely studied nutritional deficit has been lack of vitamin C, or scurvy, in which the gum is bright red, swollen, ulcerated and presents a tendency to bleed.

\section{Non-Plaque-Induced Gingival Diseases (15).}

Gingival disease of bacterial origin are those that are caused by exogenous bacterial infections produced by germs other than those that are typical components of dental plaque, such as Neisseria gonorrhoeae, Treponema pallidum, Streptococcus and other microorganisms. They manifest clinically as painful oedematous ulcerations, mucosal maculae or very swollen, non-ulcerated, atypical gums that may or may not be accompanied by lesions located elsewhere on the body. Viral gum diseases are acute manifestations of viral infections in the oral mucosa that evolve with the appearance of multiple vesicles that break easily, leading to painful ulcerations. The most important ones are associated with the herpes simplex virus (HSV) types 1 and 2 and with the varicella zoster virus. The first manifestation of HSV-1 receives the name of primary gingival stomatitis. It tends to appear in children and progresses with severe, painful gingivitis, along with the formation of blisters that go on to become fibrin-coated ulcerations. It may be accompanied by fever and lymphadenopathies. The virus reactivates in $20-40 \%$ of the cases associated with fever, trauma or ultraviolet radiation, amongst others. Small, painful clusters of blisters appear in the area of the attached gum. Chickenpox generally appears as small ulcers on the tongue, palate and gum, in addition to fever, general malaise and skin rash. The subsequent reactivation of the varicella zoster virus results in the appearance of herpes zoster, with irregular, unilateral vesiclesulcers. Fungal gingival diseases include aspergillosis, blastomycosis, candidiasis, coccidioidomycosis, criptococcosis, histoplasmosis, mucormycosis and paracoccidioidomycosis, although candidiasis and histoplasmosis are the most common. Candidiasis, predominantly caused by Candida albi- 
cans, is rarely seen in the gums of healthy individuals, although in immunocompromised subjects, it may lead to linear gingival erythema. Other forms of presentation are plaques or nodular pseudomembranous, erythematous candidiasis. Histoplasmosis is a granulomatous disease that is produced by Histoplasma capsulatum found in bird and bat faeces. They begin as nodular lesions that later become ulcerous and painful and may imitate a malignant tumour. Gingival manifestations of disorders of the skin or mucosa tend to present as erosions, vesicles, blisters, ulcers or scaly lesions. Lichen planus presents in between 0.1 and $4 \%$ of the population in two basic ways: white lichen planus and lichen planus ruber. It is characterized by white, reticular lesions, known as Wickham striae. Penfigoide is a group of disorders in which autoimmune antibodies are produced against the components of the basement membrane, causing subepithelial blisters containing clear, yellowish or bloody fluid to appear that later rupture, leading to painful, fibrin covered ulcerous lesions. In pemphigus, the autoimmune antibodies are directed against epithelial desmosomes, causing an acantholytic or intraepithelial pustule to appear that may become life threatening for the patient. Erythema multiforme is an illness producing pustules and blisters that affects both the skin and mucosa. There are two forms of the disease: minor and major (Stevens-Johnson Syndrome). Patients present swollen lips with large scabs in the area of the vermillion, although the basic lesion is a vesicle that ruptures and is followed by extensive ulcerous lesions. Lupus erythematosus is an autoimmune illness of the connective tissue in which autoimmune antibodies are directed against various cell elements, affecting the kidneys, heart, central nervous system, vascular system and bone marrow. The typical lesion consists of an atrophic central area with a whitish lacy appearance surrounded by fine striae. It is classified as discoid and systemic lupus erythematosus. Allergic reactions are not terribly common in oral mucosa, since higher concentrations of allergens are needed to provoke a reaction in the mouth as opposed to the skin. Type I immunoglobulins Emediated reactions (immediate) or Type IV T-cell mediated reactions (delayed) may develop. Traumatic lesions of the mucosa of the mouth may be accidental, iatrogenic or factitious. They may manifest as localized gingival recessions, abrasions, ulcerations or burns. They may be oedematous, erythematous or whitish in appearance or a combination of all three.

Foreign body reactions occur as a consequence of epithelial ulceration that allows foreign material to enter the gingival connective tissue. They sometimes present associated acute or chronic inflammation of the gums or they may cause tattoos. In some cases, they may become infested.

\section{Chronic Periodontitis}

The characteristic clinical signs of chronic periodontitis include loss of clinical attachment, alveolar bone loss, periodontal pocket formation and inflammation of the gums. Gingival hypertrophy or recession, bleeding on probing, increased tooth mobility and suppuration may also be associated; symptoms may even lead to tooth loss. In chronic periodontitis, the infection progresses steadily or in bursts of activity (16). Depending on its extension, it can be classified as:

- Localized, if fewer than $30 \%$ of the sites are involved.

- Generalized, if more than $30 \%$ of the sites are involved.Based on severity, it is defined as:

- Slight periodontitis: when clinical attachment loss is between 1 and $2 \mathrm{~mm}$.

- Moderate periodontitis: if attachment loss is between 3 and $4 \mathrm{~mm}$.

- Severe periodontitis: when clinical attachment loss is $5 \mathrm{~mm}$ or greater.

Currently held concepts hold that bacterial infection is the leading cause of the disease and plaque is the factor that serves as a trigger; however, defence mechanisms play a key role in its pathogenesis (Figs. 8-9).

\section{Aggressive Periodontitis}

The common features of all forms of aggressive periodontitis are: patients who, except for the presence of the periodontal infection, are otherwise clinically healthy; rapid loss of clinical attachment and bone destruction, and a positive family history (17). Other features that generally present, albeit they are not universal to all patients, are: microbial deposits that are inconsistent with the severity of tissue destruction present, high proportions of Actino-bacillus actinomycetemcomitans or Porphyromonas gingivalis, phagocytic anomalies and an abnormal hyperresponsive macrophage phenotype with high levels of prostaglandin $E_{2}$ and interleukin- $1 B$; the progression of bone loss and attachment may be dramatic (18). There are two types of aggressive periodontitis:

a. Localized. Onset is around puberty with a high antibodyresponse to infectious agents. Clinically it is characterized by interproximal attachment loss in the first molars and incisors or in at least two permanent teeth, one of which is a first molar and no more than two teeth other than first molars and incisors are involved.

b. Generalized. Generalized aggressive periodontitis tends to manifest in patients over 30 years of age, although it can appear at older ages. Antibody response is poor. There are periods during which attachment is lost, involving three permanent teeth other than first molars and incisors. 


\section{Necrotising Periodontal Diseases}

Necrotising ulcerative gingivitis (NUG) is distinguished from other gingival diseases by the presence of gingival necrosis between the teeth, with ulcerated papilla, bleeding gums and pain. This pain is the hallmark of this entity and its intensity is what leads the patient to seek treatment. Other signs and symptoms that are also associated with NUG, although not pathognomonic, include the presence of lymphadenopathies, fever, halitosis and general malaise (Fig. 10); episodes resolve within a few days following proper treatment. A series of factors exist that predispose the individual to developing this infection including stress, immunosuppression, malnutrition, smoking, trauma or pre-existing gingivitis (19). Necrotising ulcerative periodontitis (PUN) is characterized by necrosis of the gingival tissue, periodontal ligament and alveolar bone. It typically appears in subjects with systemic conditions that lead to a state of immunosuppression (20). It is possible that NUG and PUN actually represent two different states of the same infection; there is insufficient data at this point in time to be able to separate both entities into different categories. The only difference between the two is that NUG is confined to the gum, whereas PUN includes the entire attachment apparatus.

\section{Periodontal Abscesses}

A periodontal abscess is a purulent infection located in the periodontal tissues that may be a clinical manifestation in patients with moderate or severe periodontitis. The most salient features are inflammation, suppuration, reddening, extrusion of the tooth involved and the tooth is tender on percussion. The patient may at times present a low-grade fever (21) (Fig. 11 and 12). Abscesses can be classified as:

- Gingival abscess. A localized, painful and rapidly spreading lesion that affects the gingival margin or interdental papilla. It is generally an acute inflammatory response of the gingiva to a foreign body that has entered the gum.

- Periodontal abscess. A localized accumulation of pus in the gingival wall of a periodontal pocket that provokes the destruction of collagen fibre attachment and the subsequent loss of adjacent alveolar bone. It tends to be related to the presence of tortuous periodontal pockets, furcation involvement or infraosseous defects.

- Pericoronal abscess. A pericoronal abscess is a localized accumulation of pus on the gingival tissue surrounding the crown of a tooth that has not fully erupted and is generally located in the area of the third inferior molar. The gingival tissue appears red and inflamed and patients have difficulty swallowing.

\section{Periodontic-Endodontic Lesions}

Periodontal or endodontic infections may evolve with increased probing depth of the adjacent teeth, inflammation, bleeding on probing, suppuration, fistula formation, sensitivity to percussion, increased tooth mobility, angular bone loss and pain. These signs and symptoms are usually observed in plaque-induced periodontitis that begins at the gingival margin and progresses towards the root. However, they may also be the result of endodontic infections that reach the periodontal ligament through the apical foramen or by means of the lateral or accessory canals and advance towards the crown (22).

Acquired Deformities and Conditions

A number of tooth-related factors exist that may predispose the individual to the development of periodontal illnesses. Thus, although the aetiology of periodontal illnesses is bacterial, any factor that favours bacterial accumulation or allows bacteria to enter the periodontia must be taken into account (23). Mucogingival deformities, alterations of the morphology, size and interrelationships between the gum and the alveolar mucosa. This abnormality may be associated with deformities of the underlying alveolar bone (24). Occlusal trauma: damage resulting from tissue changes in the attachment apparatus as a consequence of occlusal force. Occlusal trauma may be primary, when excessive occlusal forces are exerted on a tooth with normal support; it can also be secondary, when the damage is the consequence of excessive or normal occlusal forces exerted on a tooth or teeth with diminished periodontia (25).

\section{CONCLUSION}

The revision presented here offers an overview of the different clinical syndromes encompassed within the spectrum of periodontal illnesses. Bacteria have been seen to play a leading role in the onset and subsequent development of these illnesses. Therefore, when it comes to treatment of these diseases, coadjuvant antimicrobial treatment will be needed, since we already know that scaling and root planing alone will be insufficient to eliminate the periodontal pathogens (26-29). Antimicrobial selection is made on the basis of the microbiological criteria of the illness (spectrum of action), as well as on the pharmacokinetic profile of the selected drug in particular, at all times avoiding drugrelated adverse effects $(30,31)$. The use of locally applied antimicrobial treatment enables drug levels to be attained that would not be possible by means of systemic administration; it can even make it possible to use drugs that are too toxic for systemic use (32). Nonetheless, on the other hand, systemic antibiotics exert their effect throughout the entire oral cavity and are therefore not limited to the site of application $(1,32$ 
- 34). Systemic use of amoxicillin, amoxicillin-clavulanic acid and metronidazole are recommended as the first line treatment option in aggressive periodontal illnesses. If the patient is allergic to amoxicillin or metronidazole, clindamycin, azithromycin or clarithromycin are suggested (35).

\section{BIBLIOGRAFIA / REFERENCES}

1. Mombelli A. Periodontitis as an infectious disease: specific features and their implications. Oral Dis;9:6-10.

2. Socransky SS, Haffajee AD. Dental biofilms: difficult therapeutic targets. Periodontol 2000 2002;28:12-55.

3. Marsh PD. Plaque as a biofilm: pharmacological principles of drug delivery and action in the sub- and supragingival environment. Oral Dis 2003;9:16-22.

4. Trombelli L, Tatakis DN. Periodontal diseases: current and future indications for local antimicrobial therapy. Oral Dis 2003;9:11-5.

5. Löe H, Theilade E, Jensen SB. Experimental gingivitis in man. J Periodontol 1965;36:177-87.

6. Lindhe J, Hamp SE, Löe H. Experimental periodontitis in the beagle dog. Int Dent J 1973;23:432-7.

7. Gamonal J, Bascones A, Silva A. Las quimioquinas en la patogénesis de la periodontitis. Av Periodoncia Implantol Oral 1999;11:89-95.

8. Armitage GC. Development of a classification system for periodontal diseases and conditions. Ann Periodontol 1999;4:1-6.

9. Mariotti A. Dental plaque-induced gingival diseases. Ann Periodontol 1999;4:1-19.

10. Laine, MA. Effect of pregnancy on periodontal and dental health. Acta Odontol Scand 2002;60:257-64.

11. Löe H, Silness J. Periodontal disease in pregnancy. I. Prevalence and severity. Acta Odontol Scand 1963;21:533-51.

12. Bascones A, Llanes F. Medicina Bucal. Tomo I. $2^{a}$ Edición. Madrid: Ediciones Avances; 1991. p. 274.

13. Silverstein LH, Burton CH Jr, Garnick JJ, Singh BB. The late development of oral pyogenic granuloma as a complication of pregnancy: a case report.Compend Contin Educ Dent 1996;17(2):192-8.

14. Grau DM, Silvestre FJ, Miralles L, Roig JM. La secreción salival durante el embarazo. Rev Eur Odontoestomatol 2002;XIV:93-8.

15. Holmstrup P. Non-plaque-induced gingival lesions. Ann Periodontol 1999;4:20-31.

16. Flemmig TF. Periodontitis. Ann Periodontol 1999;4:32-8.
17. Tonetti MS, Mombelli A. Early-onset periodontitis. Ann Periodontol 1999;4:39-53.

18. Darby I, Curtis M. Microbiology of periodontal disease in children and young adults. Periodontol 2000, 2001;26:3353.

19. Rowland RW. Necrotizing ulcerative gingivitis. Ann Periodontol 1999;4:65-73.

20. Novak MJ. Necrotizing ulcerative periodontitis. Ann Periodontol 1999;4:74-8.

21. Meng HX. Periodontal Abscess. Ann Periodontol 1999;4:79-83.

22. Meng HX. Periodontic-endodontic lesions. Ann Periodontol 1999;4:84-16.90.

23. Blieden TM. Tooth-related issues. Ann Periodontol 1999;4:91-7.

24. Pini Prato G. Mucogingival deformities. Ann Periodontol 1999;4:98-101.

25. Hallmon WW. Occlusal trauma: effect and impact on the periodontium. Ann Periodontol 1999;4:102-7.

26. Bascones-Martínez A, Bascones-llundain C, Campo Trapero J. Aspectos microbiológicos y control antimicrobiano de las enfermedades periodontales. RCOE 1998;3(7):657-79.

27. Falcâo Costa C, Moura e Sá A, Faria Almeida R, Bascones A. Antibioterapia en Periodoncia. Situación actual I. Antibióticos sistémicos. Av Periodon Implantol 2001;13(1):39-48.

28. Moura e Sá A, Falcâo Costa C, Faria Almeida R, Bascones A. Antibioterapia en Periodoncia. Situación actual II. Antibióticos y antimicrobianos locales. Av Periodon Implantol 2001;13:77-82.

29. Slots J. The search for effective, safe and affordable periodontal therapy. Periodontol 2000, 2002;28:9-11.

30. Dahlen G, Wikström M, Renvert S. Treatment of periodontal disease based on microbiological diagnosis. A 5 year follow-up on individual patterns. J Periodontol 1996;67:879-87.

31. Baehni PC, Takeuchi Y. Anti-plaque agents in the prevention of biofilm-as-sociated oral diseases. Oral Dis 2003;9:23-9.

32. Etienne D. Locally delivered antimicrobials for the treatment of chronic periodontitis. Oral Dis 2003;9:45-50.

33. Quirynen M, Teughels W, Van Steenberghe D. Microbial shifts after subgingival debridement and formation of bacterial resistance when combined with local or systemic antimicrobials. Oral Dis 2003;9:30-7.

34. Addy M, Martin MV. Systemic antimicrobials in the treatment of chronic periodontal diseases: a dilemma. Oral Dis 2003;9:38-44.

35. Dörfer CE. Antimicrobials for the treatment of aggressve periodontitis. Oral Dis;9:51-3. 